\title{
In Situ Morphology of the Ductus Venosus and Related Vessels in the Fetal and Neonatal Rat
}

\author{
KAZUO MOMMA, TADAHIKO ITO, AND MASAHIKO ANDO \\ Department of Pediatric Cardiology. The Heart Institute of Japan, Tokyo Women's Medical College, \\ Tokyo, Japan
}

\begin{abstract}
In situ cross-sectional morphology of the ductus venosus and related vessels was studied after rapid whole-body freezing of the fetal and neonatal rat. In the fetus, the ductus venosus was open widely, connecting the umbilical sinus and the inferior vena cava. The diameter of the ductus venosus was $50 \%$ of the diameter of the umbilical sinus. The ductus venous joined the left dorsal side of the inferior vena cava. A thin, short, membrane-like edge was present at the inner junction of the ductus venosus and the inferior vena cava, presumably effecting laminar flow of the ductus venosus blood to the left side of the thoracic inferior vena cava. A very prominent eustachian valve was present at the junction of the inferior vena cava and the right atrium, presumably directing its flow to the opening of the foramen ovale. After birth, the ductus venosus narrowed rapidly and closed completely in $2 \mathrm{~d}$. The closing ductus venosus was tubular, with the cranial end slightly wider than the caudal portion. Localized constriction was not present. These observations showed the structural substrate for preferential flow from the ductus venosus to the foramen ovale and left atrium in the fetus and did not support localized sphincter mechanism in postnatal closure of the ductus venosus. (Pediatr Res 32: 386-389, 1992)
\end{abstract}

Neonatal adaptation of the cardiovascular system has been studied mainly in fetal and neonatal lambs $(1,2)$, and the physiologic aspects have been greatly clarified $(1,2)$. Neonatal closure of the ductus venosus is one of these major adaptive changes $(1,2)$. Preferential flow of the oxygenated ductus venosus blood through the open foramen ovale to the left side of the heart was confirmed in the fetal lamb (3-5), and the anatomical basis for this preferential flow has been established (3-6). Morphologic detail of neonatal closure of the ductus venosus is more difficult to study because the ductus venosus is imbedded in the liver. Recently, we developed a method to study the in situ morphology of the cardiovascular system of the fetal and neonatal rat without postmortem changes (7-10), and morphologic details of the ductus venosus and related vessels in the fetal and neonatal rat are reported in this study.

\section{MATERIALS AND METHODS}

Animals. Twenty virgin Wistar rats (pregnancy period $21.5 \mathrm{~d}$ ) were mated overnight from 1700 to $0900 \mathrm{~h}$, and the presence of

Received February 21, 1992; accepted May 20, 1992.

Correspondence and reprint requests: Kazuo Momma, M.D. The Heart Institute of Japan, Tokyo Women's Medical College, Kawadacho 8-1, Shinjuku-ku, Tokyo, 162. Japan.

Supported by a grant-in-aid from the Ministry of Education, Science, and Culture of Japan and by the Japan Research Promotion Society for Cardiovascular Diseases. sperm in vaginal smears fixed the zero day of pregnancy. Rats were fed commercial solid food and water. Average litter size was 13. Treatment of the rats conformed to the guiding principles of the American Physiological Society.

Freezing, cutting, and photographing. Fetal and neonatal vascular morphology were studied using the rapid whole-body freezing technique, as previously reported $(7-10)$. For fetal studies, four pregnant rats were killed on the $21 \mathrm{st} d$ by cervical dislocation and frozen immediately in liquid nitrogen. Thereafter, frozen fetuses were removed. In the study of newborn rats, 14 mother rats nursed newborns for $1,2,3$, or $4 \mathrm{~d}$, after which these newborns were frozen in dry ice-acetone. For the study of the neonate immediately after birth, fetuses were delivered by cesarean section from two rats on the 21 st $\mathrm{d}$ and were frozen immediately, or after survival for 3 to $8 \mathrm{~h}$ at $37^{\circ} \mathrm{C}$, in dry ice-acetone.

Frozen lower thoraxes including upper abdomen were trimmed and sectioned on a freezing microtome (Komatsu Solidate Co., Tokyo, Japan) in the frontal, transverse, sagittal, or cardiac short-axis plane. Cross-sections were photographed with a binocular stereoscopic microscope (Wild M 400 Photomakroscope, Wild Heerbrugg Ltd., Heerbrugg, Switzerland) using color film (Reala, Fuji Film Co., Tokyo, Japan). Cross-sections of the lower thorax and upper abdomen were photographed serially every $200 \mu \mathrm{m}$, including the umbilical sinus, the ductus venosus, the inferior vena cava, the right atrium, and the interatrial septum. In each rat, about 15 cross-sections were photographed. Numbered section paper $(1 \times 1 \mathrm{~mm})$ was photographed for scale, and the pictures were printed on paper.

Measurement. Overall pictures of the ductus venosus and the umbilical sinus were obtained in sagittal section. The eustachian valve at the junction of the inferior vena cava and the right atrium was clearly visualized in cardiac short-axis plane. The maximum diameters of the umbilical sinus in the fetus and the portal sinus in the neonatal rat were measured in the frontal section. The caudal and cranial inner diameters of the ductus venosus were measured in the frontal section. The sinus portion connecting the umbilical vein and the ductus venosus is termed umbilical sinus in this article, and the same part became the anterior portion of the neonatal portal sinus.

Statistical analysis. Morphometric data are presented as mean \pm SEM. Comparisons between the two groups were subsequently submitted to $t$ test at a confidence level of $95 \%$.

\section{RESULTS}

In the fetus, the umbilical vein continued to the umbilical sinus, which joined the ductus venosus and the portal vein (portal sinus) (Figs. 1 and 2). The ductus venosus coursed craniodorsally and connected to the left side of the inferior vena cava (Figs. 1 and 2). The diameter of the ductus venosus was consistently larger in the cranial than in the caudal part (Fig. 1). 


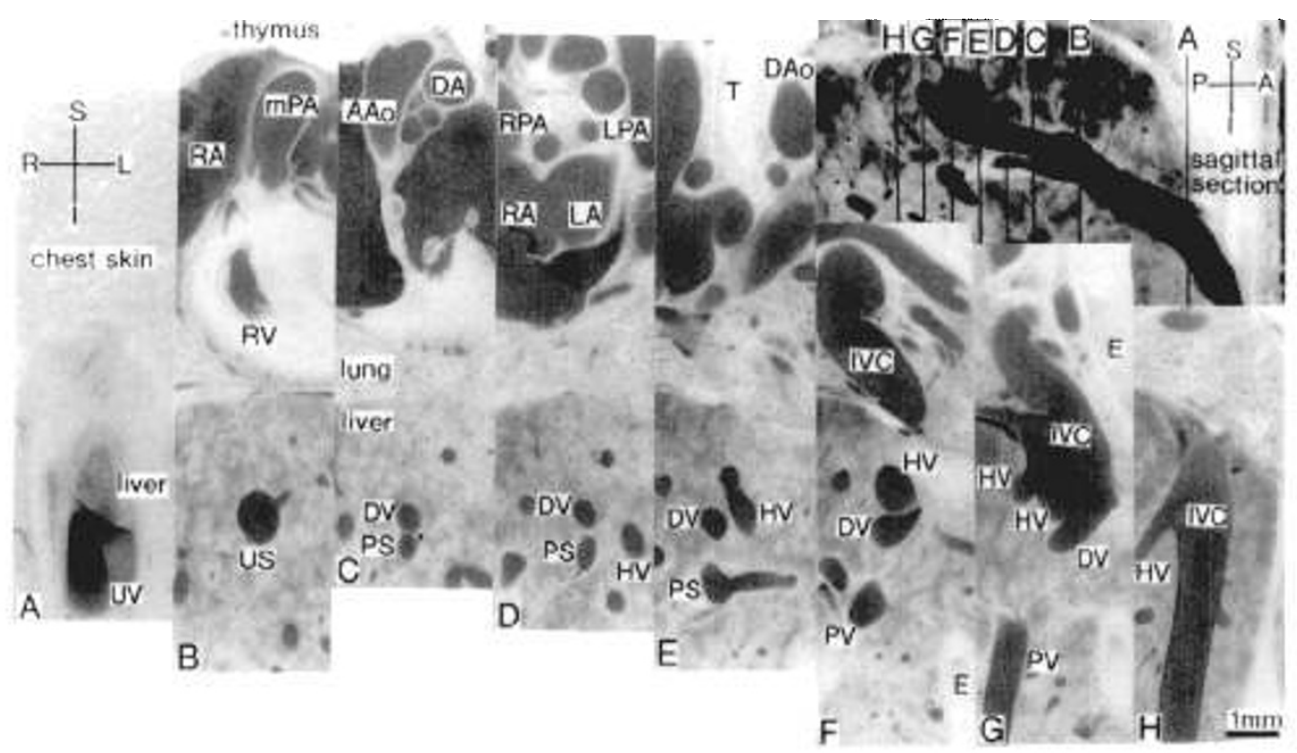

Fig. 1. Fetal umbilical vein and ductus venosus in the frontal plane $(A-H)$. The position of each frontal plane is shown in the right sagittal picture at the right upper corner. A large umbilical vein $(A)$ continued to the umbilical sinus $(B)$. The umbilical sinus connected with the ductus venosus and the portal sinus or portal vein $(C)$. The ductus venosus coursed dorsally and cranially $(D$ and $E)$ and joined the inferior vena cava after connection to the left hepatic vein $(F)$ at its left side $(G)$. Abbreviations: $A$, anterior; $D A$, ductus arteriosus; $D A o$, descending aorta; $D V$, ductus venosus; $E$, esophagus; $H V$, hepatic vein; $I$, inferior; $I V C$, inferior vena cava; $L$, left; $L A$, left atrium; $L P A$, left pulmonary artery; $m P A$, main pulmonary artery; $P$, posterior; $P S$, portal sinus; $P V$, portal vein; $R$, right; $R A$, right atrium; $R P A$, right pulmonary artery; $R V$, right ventricle; $S$, superior; $T$, trachea; $U S$, umbilical sinus; and $U V$, umbilical vein.
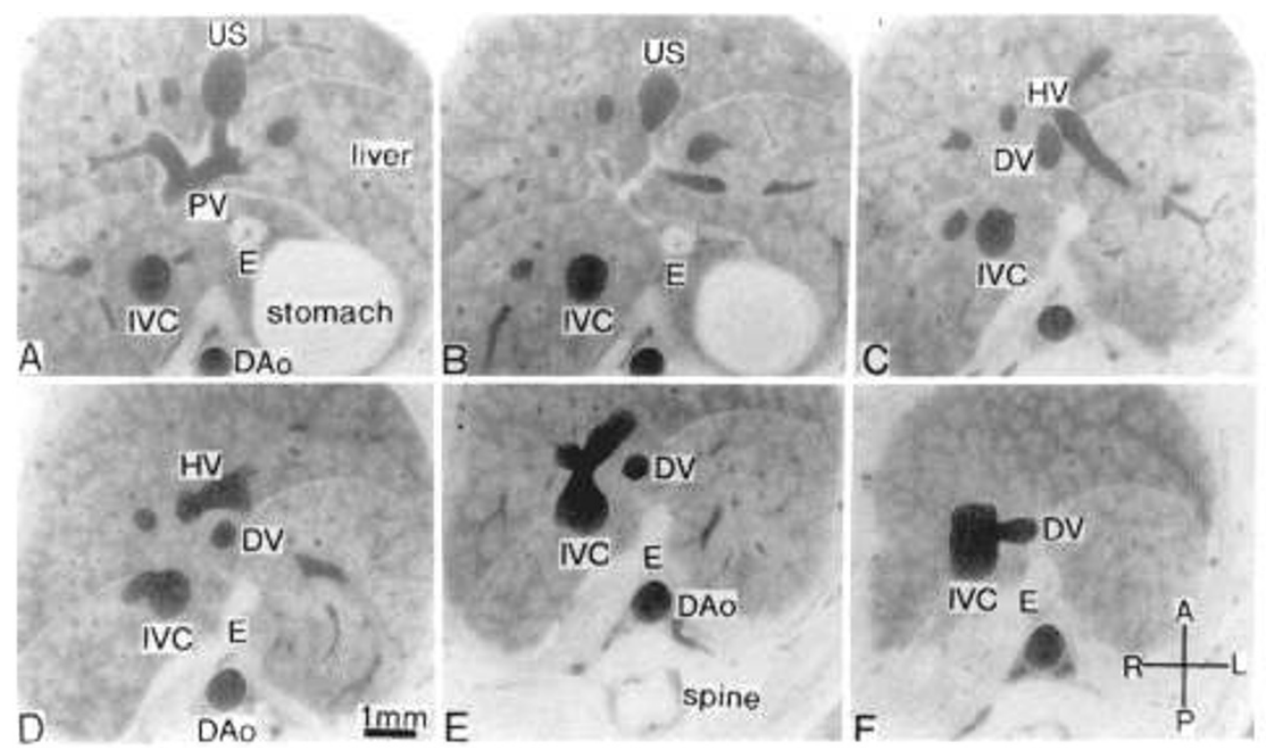

Fig. 2. Fetal umbilical sinus and ductus venosus in the transverse plane. In the caudal plane, the umbilical sinus joins with the portal vein $(A)$. The ductus venosus bifurcates from the craniodorsal part of the umbilical sinus $(B$ and $C)$ and courses dorsocranially $(D$ and $E)$ until it joins to the left side of the inferior vena cava $(F)$. Abbreviations are the same as in Figure 1.

In the neonate, the diameter of the ductus venosus became smaller rather uniformly (Fig. 3), and it closed $2 \mathrm{~d}$ after birth (Figs. 4 and 5). The inner diameter of the closing ductus venosus was slightly larger in the cranial than in the caudal part (Figs. 3 and 5). Localized narrowing was not present at the junction with the portal sinus (Fig. 3). The portal sinus rapidly became smaller $1 \mathrm{~d}$ after birth (Fig. 5).

A prominent eustachian valve was consistently present at the junction of the inferior vena cava and the right atrium in the fetus (Fig. 6). In the short axis section, the eustachian valve opened in the direction of the opening of the foramen ovale and reached halfway to the crista dividens of the foramen ovale (Fig. 6).

\section{DISCUSSION}

Our study reveals the time course and morphologic details of the closure process of the neonatal ductus venosus in the rat. The time course of the closure of the human ductus venosus has been of interest because of its use in catheterizing neonates (11, 12) and occasional reports of its patency in later life (13) but has never been clarified. Meyer and Lind (14) stated that the human ductus venosus closes functionally soon after birth, and its anatomical closure occurs in 15 to $20 \mathrm{~d}$ after birth. Linde et al. (12) reported catheter passage from the umbilical vein through the ductus venosus in $64 \%$ of neonates less than $6 \mathrm{~d}$ of age and in none beyond that age. Sapin et al. (11) showed an angiogram in 


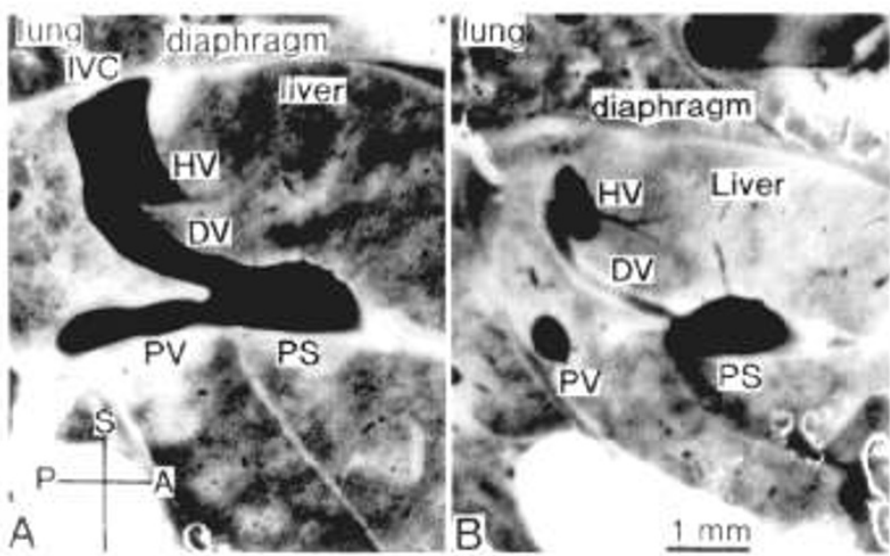

Fig. 3. Sagittal section of the neonatal ductus venosus at $3 \mathrm{~h}(A)$ and $8 \mathrm{~h}(B)$ after birth. The ductus venosus bifurcates from the portal sinus and courses craniodorsally. No narrowing is obvious at $3 \mathrm{~h}(A)$. The ductus venosus is narrow tubularly at its total length at $8 \mathrm{~h}(B)$. The cranial end is wider than the caudal part $(B)$. No localized narrowing is obvious at the junction with the portal sinus at 3 or $8 \mathrm{~h}$. Abbreviations are the same as in Figure 1.

an 8-d-old neonate with an open ductus venosus that could not be passed with a catheter. These clinical findings offer support that the human ductus venosus closes functionally several days after birth. However, a closing ductus venosus may be opened by a catheter or increased pressure associated with injection of contrast material, so these findings may overestimate its patency.

Because of difficulty in determination of ductus venosus closure in humans, the time course of its closure has been investigated in the lamb. Zink and Van Petten (15) studied the time course of closure of the ductus venosus in the newborn lamb with microsphere techniques. In their study, closure occurred within $48 \mathrm{~h}$ after birth in most cases. Botti et al. (16) also studied the time course in the newborn lamb and showed open ductus venosus shunting from the portal vein to the inferior vena cava up to $6 \mathrm{~d}$ after birth. In both studies, large variations were present, and it was difficult to correlate results with actual size of the ductus venosus.
Our present study demonstrates clearly the morphology of the closing ductus venosus in the neonatal rat with a rapid wholebody freezing method, enabling study of in situ vascular morphology without postmortem changes (17). In our observations, closure of the ductus venosus was completed $2 \mathrm{~d}$ after birth. The closing ductus venosus showed tubular narrowing and no localized constriction at the junction with the portal sinus.

Several mechanisms have been proposed for postnatal closure of the ductus venosus. A sphincter mechanism at the junction with the portal sinus was proposed as an active constriction inducing its closure (2). Our observations do not support the presence of a localized sphincter at the junction with the portal sinus. Although in vitro study revealed relaxation with prostaglandin $E_{2}$ and $I_{2}$ and constriction with indomethacin in the ductus venosus of the fetal lamb (18), the in situ fetal ductus venosus was rather unresponsive to several stimuli such as hypoxia (3) and nonsteroidal antiinflammatory drugs (19). The mechanism of postnatal closure of the ductus venosus remains to be clarified.

Physiologic studies in the lamb have established a preferential flow from the umbilical vein to the left side of the heart in the fetus (3-6). These studies showed the connection of the ductus venosus to the left dorsal portion of the inferior vena cava, a semivalve at the junction of the ductus venosus and the inferior vena cava, and dorsal position of the opening of the inferior vena cava facing the foramen ovale (3-6). Our present study confirms these earlier observations. In addition, the present study shows a well-developed eustachian valve at the junction of the inferior vena cava and the right atrium, presumably directing blood from the inferior vena cava through the foramen ovale to the left atrium. Elias and Perry (20) reported that in the human fetus the ductus venosus joined the left side of the inferior vena cava. The eustachian valve, although less developed, was present in more than $70 \%$ of human hearts $(21)$. This suggests that preferential flow of umbilical venous blood to the brain and heart is also present in the human fetus.

Acknowledgment. The authors thank Leonard M. Linde, Professor of Pediatric Cardiology, University of Southern California School of Medicine, for his editorial help.
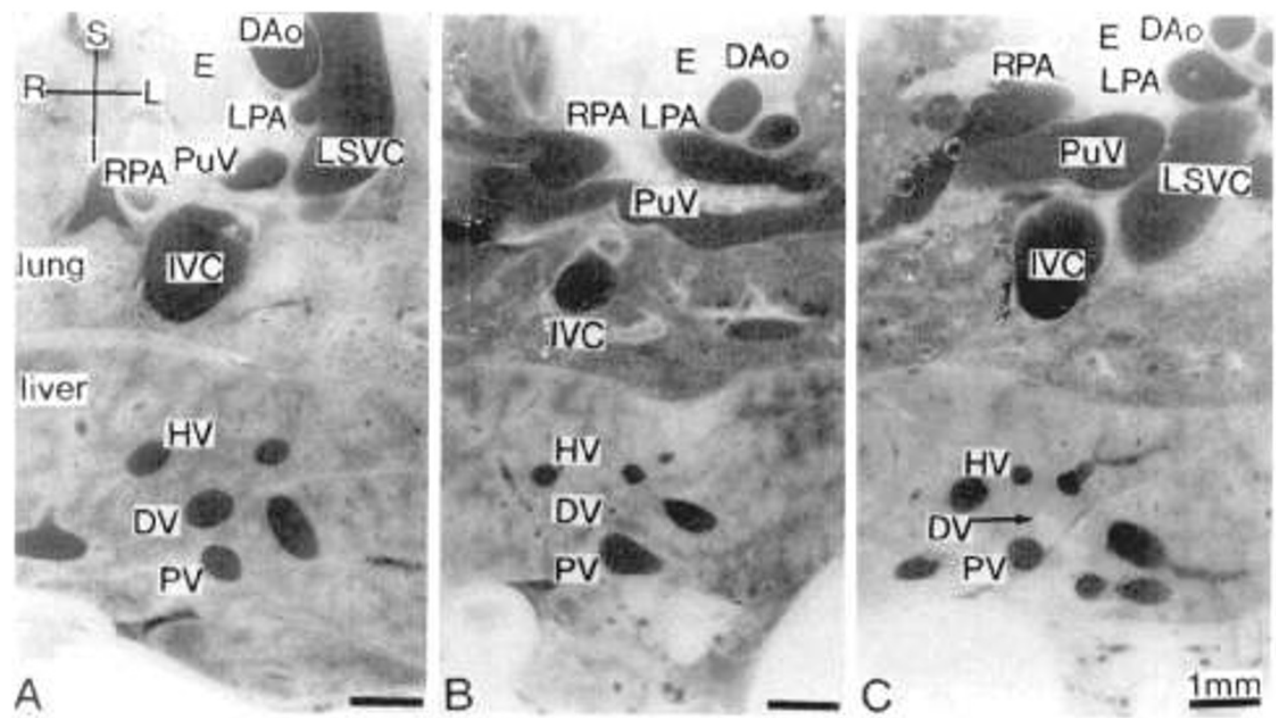

Fig. 4. Neonatal closing ductus venosus in the frontal section. In the fetus, the ductus venosus is widely open $(A)$. It is much smaller with a white. thick wall $1 \mathrm{~d}$ after birth $(B)$, and it is closed completely at $2 \mathrm{~d}(C)$. Abbreviations: $L S V C$, left superior vena cava; $P u V$, pulmonary vein; others are the same as in Figure 1. 


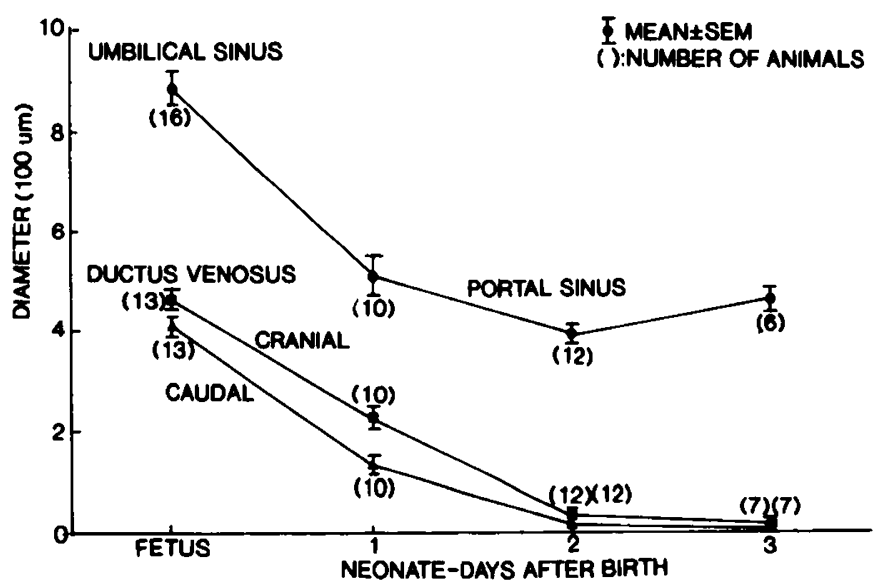

Fig. 5. Neonatal change of the inner diameter of the ductus venosus and portal sinus in the rat.

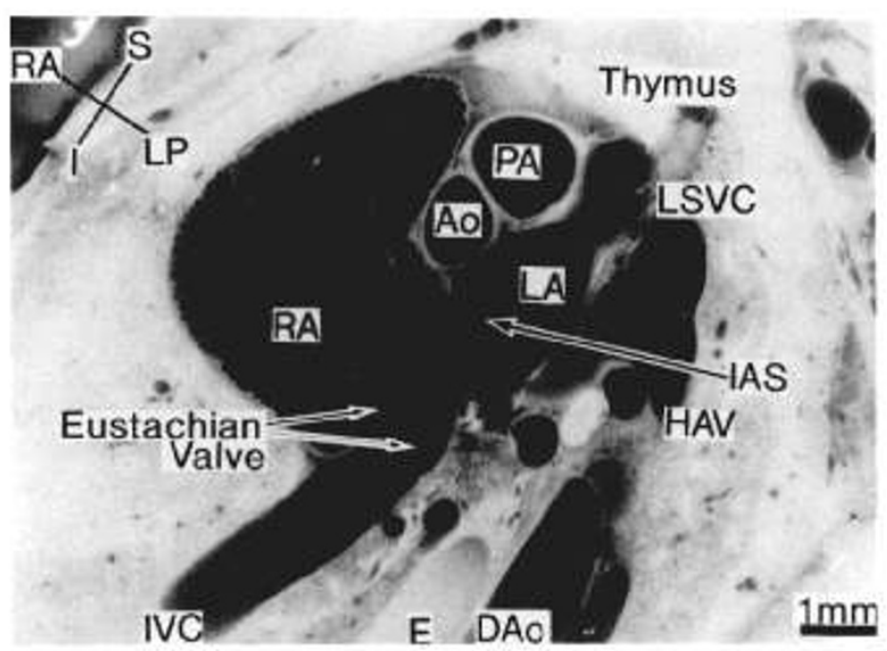

Fig. 6. The inferior vena cava, the eustachian valve, and the foramen ovale of the fetal rat in the cardiac short-axis plane. Abbreviations: $A O$, aorta; $L P$, left posterior; $L S V C$, left superior vena cava; $H A V$, hemiazygos vein; $I A S$, interatrial septum; $P A$, pulmonary artery; others are the same as in Figure 1.

\section{REFERENCES}

1. Dawes GS 1968 Foetal and Neonatal Physiology. Year Book Medical Publishers, Chicago

2. Rudolph AM 1974 Congenital Diseases of the Heart. Year Book Medical Publishers, Chicago

3. Edelstone DI, Rudolph AM 1979 Preferential streaming of ductus venosus blood to the brain and heart in fetal Lambs. Am J Physiol 273:H724-H729

4. Bristow J, Rudolph AM, Itskovitz J 1981 A preparation for studying liver blood flow, oxygen consumption, and metabolism in the fetal lamb. J Dev Physiol 3:255-266

5. Rudolph AM 1983 Hepatic and ductus venosus blood flows during fetal life. Hepatology 3:254-258

6. Rudolph AM 1986 Fetal cardiovascular shunts: their role in oxygen and substrate delivery. In: Johansen K, Burggren W (eds) Cardiovascular Shunts. Raven Press, New York, pp 243-254

7. Momma K, Takao A, Ito R, Nishikawa T 1987 In situ morphology of the heart and great vessels in fetal and newborn rats. Pediatr Res 22:573-580

8. Momma K, Takao A 1990 Right ventricular concentric hypertrophy and left ventricular dilatation by ductal constriction in fetal rats. Circ Res 64:11371146

9. Momma K, Ando M, Takao A 1990 Fetal cardiac morphology of tetralogy of Fallot with absent pulmonary valve in the rat. Circulation 82:1343-135

10. Momma K, Konishi H, Hagiwara H 1985 Characteristic morphology of the constricted fetal ductus arteriosus following maternal administration of indomethacin. Pediatr Res 19:493-500

11. Sapin SO, Linde LM, Emmanouilides GC 1963 Umbilical vessel angiocardiography in the newborn infant. Pediatrics 31:946-951

12. Linde LM, Higashino SM, Berman G, Sapin SO, Emmanouilides GC 1966 Umbilical vessel cardiac catheterization and angiocardiography. Circulation 34:984-988

13. Michell IM, Pollock JCS, Gibson AAM 1991 Patent ductus venosus. Pediatr Cardiol 12:181-183

14. Meyer WW, Lind J 1966 The ductus venosus and the mechanism of its closure. Arch Dis Child 41:597-605

15. Zink J, Van Petten GR 1980 Time course of closure of the ductus venosus in the newborn lamb. Pediatr Res 14:1-3

16. Botti JJ, Edelstone DI, Caritis SN, Mueller-Heubach E 1982 Portal venous blood flow distribution to liver and ductus venosus in newborn lamb. Am J Obstet Gynecol 144:303-308

17. Hoernblad PY, Larsson KS 1967 Studies on closure of the ductus arteriosus I. Whole-body freezing as improvement of fixation procedure. Cardiologia 51:231-240

18. Adeagbo ASO, Coceani F, Olley PM 1982 The response of the lamb ductus venosus to prostaglandins and inhibitors of prostaglandin and thromboxane synthesis. Circ Res 51:580-586

19. Momma K, Takeuchi H, Hagiwara H 1984 Pharmacological constriction of the ductus arteriosus and ductus venosus in the fetal rat. In: Nora JJ, Takao A (eds) Congenital Heart Disease. Causes and Processes. Futura, Mount Kisco, NY, pp 313-327

20. Elias H, Perry D 1952 Gross anatomy of the blood vessels and ducts within the human liver. Am J Anat 90:59-111

21. Theore DG, Ursell PC, Ho SY, Smith A, Bowman FO, Gersony WM, Anderson RH 1991 Atypical morphologic features in tricuspid atresia. J Thorac Cardiovasc Surg 102:606-610 